\title{
Erratum to: On the second weight of generalized Reed-Muller codes
}

\author{
Olav Geil
}

Published online: 24 April 2014

(C) Springer Science+Business Media New York 2014

\section{Erratum to: Des. Codes Cryptogr. (2008) 48:323-330 DOI 10.1007/s10623-008-9211-9}

Theorem 3 of the above paper is stated wrongly. It should be as follows:

Theorem 3 Let $s, m$ be integers with $2 \leq s<q$ and $2 \leq m$. The second weight of $R M_{q}(s, m)$ is equal to $q^{m}-s q^{m-1}+(s-1) q^{m-2}$. There exist codewords with Hamming weight equal to the second weight that are defined from products of linear factors.

The online version of the original article can be found under doi:10.1007/s10623-008-9211-9.

O. Geil $(\bowtie)$

Department of Mathematical Sciences, Aalborg University, Aalborg, Denmark e-mail: olav@math.aau.dk 